\title{
Fibrina rica en plaquetas (FRP): Una alternativa terapéutica en odontología
}

\author{
Platelet rich fibrin (PRF): A therapeutic alternative in dentistry \\ Wilfredo Escalante Otárola 1,a, Gabriela Castro Núñez ${ }^{1, a}$, Luis Geraldo Vaz ${ }^{1, b}$, Milton Carlos Kuga 1,b
}

\begin{abstract}
RESUMEN
El objetivo de esta revisión, fue describir de una manera sencilla las principales características de la Fibrina Rica en Plaquetas (FRP), su composición, propiedades y aplicación clínica. La FRP, biomaterial autógeno y concentrado plaquetario de segunda generación, es una matriz de fibrina que contiene leucocitos, plaquetas y factores de crecimiento, que son necesarios para los procesos de cicatrización, lo que brinda a este biomaterial, una gran utilidad en diversas áreas de la salud, incluyendo la odontología. Con esta revisión concluimos que la FRP es una alternativa real para mejorar la cicatrización de procedimientos quirúrgicos y potenciar otros biomateriales regenerativos en diversas áreas de la odontología, además de su accesibilidad y bajo costo.
\end{abstract}

PALABRAS CLAVE: Concentrados plaquetario, fibrina rica en plaquetas, proceso de cicatrización

\section{SUMMARY}

The aim of this review was to describe in a simple way the main characteristics of platelet rich Fibrin (PRF), its composition, properties and clinical application. PRF, biomaterial autologous and platelet concentrate of second generation, is a fibrin matrix containing leukocytes, platelets and growth factors, which are necessary for the healing process, giving to this biomaterial, very useful in various areas of health, including dentistry. With this review, we conclude that FRP is a real alternative to improve healing of surgical procedures and enhance other regenerative biomaterials in several areas of dentistry, in addition to accessibility and low cost.

\section{KEYWORDS: platelet concentrates, platelet rich fibrin, healing process.}

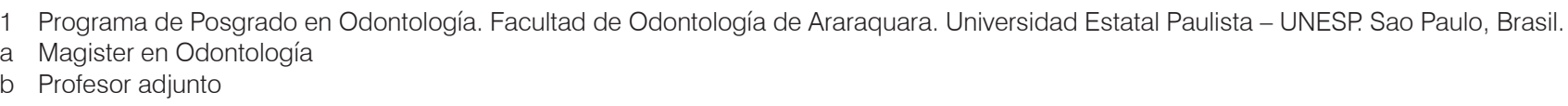




\section{INTRODUCCIÓN}

Los biomateriales son materiales orgánicos o sintéticos utilizados en contacto con sistemas biológicos cuya finalidad es la de reparar o sustituir tejidos, órganos o funciones del organismo, para mantener o mejorar la calidad de vida del paciente. Siendo así, para utilizar un biomaterial con seguridad, este debe presentar tres características básicas: (I) biocompatibilidad, no induciendo respuestas biológicas adversas, como reacciones alérgicas e inflamatorias no tolerables por el organismo; (II) alta conductividad, estimulando el crecimiento de células; (III) bioactividad, que es la capacidad del material para unirse con tejido biológico (1).

Los biomateriales pueden ser clasificados de acuerdo con su origen, siendo biológicos (autógenos - del mismo paciente; alógenos - obtenidos de un donador; o exógenos - obtenidos de algún animal) o sintéticos/ aloplásticos (metales, cerámicos y polímeros); o por la respuesta inducida al medio biológico, pudiendo ser bioinertes, bioabsorvibles y bioactivos. Los materiales bioactivos poseen la capacidad de interactuar íntimamente con el tejido biológico (bioadhesión), a diferencia de los materiales bioinertes y bioabsorvibles, donde la respuesta inducida por estos se da por medio de la formación de una capa de tejido fibroso entre el material y el tejido biológico, imposibilitando así la interacción directa entre material y tejido, lo que podrá resultar en inestabilidades y fallas (1). El desarrollo de los aditivos quirúrgicos bioactivos es uno de los mayores desafíos de la investigación clínica, los que han sido usados para regular la inflamación y aumentar la velocidad del proceso de cura (2). Sin embargo, se sabe que las plaquetas desempeñan un papel crucial, no apenas en la homeostasis, sino también en el proceso de cicatrización de la herida (3).

En 1974, el potencial regenerativo de las plaquetas fue introducido por Ross et al. (4), quienes fueron los primeros en describir un factor de crecimiento a partir de plaquetas. Las plaquetas liberan factores de crecimiento que están presos en el interior de la matriz de fibrina después de su activación. Estos factores de crecimiento son considerados como estimulantes para la respuesta mitogénica en el periostio y son responsables por la reparación del hueso durante la cicatrización normal de las heridas (5). En un esfuerzo para superar restricciones legales sobre la manipulación de sangre con plasma rico en plaquetas, una nueva familia de concentrados plaquetarios fue contemplada para desarrollar, lo que vino a ser reconocido como, fibrina rica en plaquetas (FRP) (2).

La FRP de Choukroun (2) es una matriz de fibrina, considera un material autógeno y bioactivo que fue inicialmente utilizado en la implantología oral (6). Actualmente, varias investigaciones muestran su aplicación en diversas disciplinas de la odontología (7-12).

\section{REVISIÓN DE LITERATURA}

\section{Proceso de cicatrización}

El proceso de cicatrización ocurre para reponer la integridad de los tejidos lacerados, como una reacción de defensa del organismo frente a un agente traumático, siendo la misma, descrita como un proceso biológico y complejo, teniendo etapas clasificadas como: inflamación, proliferación y remodelación/reparación (13).

La cicatrización ocurre con una variedad de eventos intra y extracelulares, regulados por proteínas de señalización, siendo un proceso incompleto; a pesar de esto, se describe que las plaquetas actúan no solo en la hemostasia, sino también en el proceso de cicatrización de la herida (3). Normalmente las plaquetas son atraídas para el local de la herida, estimulando la formación de fibrina y la cascada de coagulación (14). Además, varios estudios han demostrado que los factores de crecimiento están presentes en cada una de estas fases, estimulando la angiogénesis y osteogénesis, la inducción de la quimiotaxis, la proliferación y diferenciación de células progenitoras para el local de la herida, promoviendo la mitosis celular y la síntesis de colágeno. Es por eso que en el intento de mejorar la calidad del coagulo, fueron desarrollados los concentrados plaquetario.

\section{Evolución de los concentrados plaquetarios}

Los concentrados plaquetarios son biomateriales autógenos obtenidos por citaféresis, para separar las plaquetas. Las transfusiones de plaquetas son usadas para tratar o prevenir hemorragias en pacientes con trombocitopenia, leucemia u otros trastornos plaquetarios. En las últimas dos décadas, hubo una mejor comprensión de las propiedades fisiológicas de las plaquetas en la reparación de heridas, lo que llevo al aumento de sus aplicaciones terapéuticas en formas diferentes y con resultados variables (15). Por 
tal motivo, los concentrados plaquetarios tienen una fuerte base científica y biológica, tornándose alternativas terapéuticas a disposición. Estos concentrados son básicamente clasificados como de: $1^{\circ}$ generación - Plasma rico en Plaqueta (PRP), obtenido a través de dos tiempos de centrifugación, pero con la adición de un anticoagulante antes de la primera y trombina bovina después de la segunda; y de $2^{\circ}$ generación Fibrina rica en plaquetas (FRP), obtenida a través de un tiempo de centrifugación y sin aditivos (2).

En 1915, El Dr. Grey fue el primero en usar fibrina de la sangre para el control del sangramiento en una cirugía cerebral (16). El Dr. Choukroun, médico anestesiólogo dedicado al tratamiento del dolor, en el 2000 propuso el protocolo de FRP para cicatrización de heridas de difícil reparación y para tratamiento del dolor crónico, y en el 2006 introdujo el uso de FRP para tratamientos odontológicos (2).

\section{Fibrina rica en plaquetas}

La fibrina rica en plaquetas (FRP) es un concentrado plaquetario de segunda generación, obtenido como una membrana de fibrina, con alto potencial de regeneración tecidual. Las plaquetas contenidas en la FRP liberan factores de crecimiento que optimizan el proceso de regeneración, además la matriz de fibrina promueve la angiogénesis, facilitando el acceso al local lesionado, desempeñando un importante papel en la cicatrización tecidual (2). El proceso de obtención de la FRP es considerado simple y de bajo costo. Estos concentrados plaquetarios proporcionan alternativas terapéuticas, utilizando material autógeno con potencial para estimular el proceso fisiológico de la cicatrización, y auxiliar en la regeneración de diversos tejidos.

\section{Proceso de obtención de FRP}

La FRP fue desarrollada por primera vez en Francia por Choukroun et. al. (2006) para uso específico en cirugía oral y maxilofacial. Esta técnica no exige anticoagulante, ni trombina bovina (u otro agente de gelificación), siendo solo sangre centrifugada, sin aditivos, motivo que hizo posible evitar todas las restricciones de la ley francesa sobre la recolocación de productos derivados de la sangre. La obtención es realizada a través del depósito de una muestra de sangre en tubos de ensayo, sin anticoagulante, para ser centrifugada instantáneamente. Una centrifuga de mesa puede ser usada para este fin durante 12 minutos a $2700 \mathrm{rpm}$ (2). El producto resultante es compuesto de tres fases: una fase superior de plasma acelular pobre en plaquetas (PPP) de color amarillento; en una fase intermedia correspondiente al coagulo de FRP; y una fase inferior rojiza, correspondiente a los glóbulos rojos (hematíes).

La activación de la mayoría de las plaquetas de la muestra de sangre ocurre cuando entran en contacto con la superficie del tubo de ensayo y comienza después de algunos minutos el proceso de la cascada de coagulación. El coagulo de fibrina es obtenido en la mitad del tubo, entre los glóbulos rojos del fondo y el plasma acelular de la parte superior. Gran número de plaquetas, quedan presas en las mallas de fibrina (17).

\section{Composición de la FRP}

Fibrina: es la forma activa de una molécula plasmática llamada fibrinógeno. Esta molécula fibrilar soluble está masivamente presente en el plasma y en los gránulos alfa de las plaquetas, y desempeña un papel importante en la agregación plaquetaria durante la hemostasia. Se convierte en un tipo de pegamento biológico capaz de consolidar el grupo inicial de plaquetas, que es una pared de protección a lo largo de rupturas vasculares durante la coagulación. El fibrinógeno es el substrato final, para todas las reacciones de coagulación, siendo una proteína soluble. El fibrinógeno es convertido en fibrina insoluble a través de la trombina, mientras que el gel de fibrina polimerizada es la primera matriz cicatricial de la herida (2).

Leucocitos: son células sanguíneas heterogéneas, móviles, de morfología esferoidal y que son encontradas transitoriamente en la sangre, nacen en la medula ósea y en el tejido linfático. Defienden al organismo, actuando sobre el sistema inmunológico (2).

Plaquetas: son células sanguíneas anucleadas, que en su citoplasma contienen numerosos gránulos alfa, que son los que almacenan los factores de crecimiento. Con la activación de las plaquetas, empieza la agregación plaquetaria, donde los gránulos alfa liberan leucocitos y factores de crecimiento, correspondiendo a los elementos más importantes en los procesos de cura y reparación (2). Estos factores de crecimiento tienen naturaleza proteica y su importancia, en los procesos de cicatrización y reparo, radica en su capacidad para modificar respuestas biológicas, regulando procesos como migración, proliferación, 
diferenciación y metabolismo (18).

PDGF (factor de crecimiento derivado de plaquetas), actúa en la reparación y proliferación celular. Su actividad mitogénica estimula la quimiotaxis de monocitos y macrófagos, fagocitosis de monocitos y neutrófilos y síntesis del colágeno $(19,20,21)$.

VEGF (factor de crecimiento endotelial vascular), mitógeno selectivo de células endoteliales con acción angiogénica in vivo

TGF-beta (factor de crecimiento transformador beta), mejora la deposición de la matriz extracelular, aumentando su síntesis e inhibiendo la degradación de colágeno (22).

IGF-I (factor de crecimiento insulínico tipo I), es el más abundante en el tejido óseo, es producido por osteoblastos y estimula la formación de hueso induciendo la proliferación celular, diferenciación y la biosíntesis de colágenos tipo I (23); también es encontrado en cantidades significativas en las plaquetas. Cuando es liberado por estas últimas, es un factor quimiotáctico poderoso para células endoteliales vasculares, causando un aumento en la neovascularización de la herida.

EGF (factor de crecimiento epidérmico), los niveles en plasma son indetectables pero en las plaquetas son encontrados en cantidades apreciables. Después de la activación de las plaquetas, es liberada una cantidad suficiente para inducir la migración y mitosis celular (19).

\section{Aplicaciones clínicas de la FRP}

Cada vez existen más relatos sobre las aplicaciones clínicas de la FRP en diferentes disciplinas de la odontología, sin embargo aún no existen estudios concluyentes sobre los notables beneficios que presenta. Podemos encontrar relatos de casos donde se obtuvieron resultados satisfactorios al utilizar la FRP como una alternativa disponible, que principalmente mejora la calidad de la cicatrización y puede potenciar otros biomateriales regeneradores. Entre las aplicaciones más recientes encontradas en la literatura, se describe que puede ser utilizada en cirugías de elevación del seno maxilar, como protector y/o reparador de la membrana sinusal $(24,25)$, en cirugías de colocación de implantes, puede servir como una membrana adicional para mejorar la calidad de la cicatrización (26), en preservación de rebordes alveolares postexo- doncia, para mejorar la cicatrización, disminuir el riesgo de infección y de dolor postoperatorio (27), en cirugías de aumento óseo utilizando mallas de titanio, para mejorar el cierre por primera intensión (28), como membrana en combinación con técnica de colgajo reposicionado lateralmente y otros tratamientos de recesiones gingivales $(29,30)$, en combinación con injerto óseo y otros biomateriales regeneradores para el tratamiento de lesiones de furca endo-periodontales (31,32), como relleno en cirugías paraendodónticas, en el tratamiento de lesiones periapicales $(33,34)$, en tratamientos de revascularización de dientes permanentes inmaduros con necrosis pulpar (35), en tratamiento de perforaciones de piso pulpar en región de furca (36), en combinación con MTA en tratamiento de revitalización de dientes inmaduros con necrosis pulpar (37) y como barrera apical (38), en tratamientos de osteonecrosis alveolar (39), entre otros.

\section{CONCLUSIONES}

La FRP es un material bioactivo con beneficios regenerativos evidentes, sin embargo el respaldo científico de sus beneficios aun es limitado. La FRP pertenece a una segunda generación de concentrados plaquetarios, siendo más rápida y fácil su obtención, sin necesidad de aditivos, haciendo de este concentrado un material más seguro. La actividad biológica de la molécula de fibrina, es por si sola suficiente para explicar la capacidad de cicatrización de la FRP. Además de sus beneficios, su bajo costo hace de este biomaterial autógeno, una buena alternativa terapéutica en odontología y que en combinación con otros biomateriales, puede mejorar las propiedades regeneradoras de estos.

\section{Correspondencia:}

Wilfredo Escalante Otárola

Email: wilfredogeotarola@foar.unesp.br

\section{REFERENCIAS BIBLIOGRAFICAS}

1. Guastaldi AC, Aparecida AH. Calcium phosphates of biological interest: Importance as biomaterials, properties and methods for coatings obtaining. Quim Nova. 2010;33(6):1352-8.

2. Dohan DM, Choukroun J, Diss A, et al. Platelet-rich fibrin (PRF): A second-generation platelet concentrate; Part I: Technological concepts and evolution. Oral Surg Oral Med Oral Pathol Oral Radiol Endod. 2006; 101: e37-44. 
3. Gassling VL, Açil Y, Springer IN, Hubert N, Wiltfang J. Platelet-rich plasma and platelet-rich fibrin in human cell culture. Oral Surg Oral Med Oral Pathol Oral Radiol Endod. 2009; 108:48-55.

4. Ross R, Glomset J, Kariya B, Harker L. A plateletdependent serum factor that stimulates the proliferation of arterial smooth muscle cells in vitro. Proc Natl Acad Sci USA. 1974; 71:1207-10.

5. Khiste SV, Tari RN. Platelet-rich fibrin as a biofuel for tissue regeneration. ISRN Biomaterials. 2013; 2013:1-6. doi: http://dx.doi.org/10.5402/2013/627367

6. Aroca S, Keglevich T, Barbieri B, Gera I, Etienne D. Clinical evaluation of a modified coronally advanced flap alone or in combination with a platelet-rich fibrin membrane for the treatment of adjacent multiple gingival recessions: a 6-month study. J Periodontol. 2009; 80:244-252.

7. Arunachalam LT, Merugu S, Sudhakar U. A novel surgical procedure for papilla reconstruction using platelet rich fibrin. Contemp Clin Dent. 2012; 3:467-470.

8. Bansal C, Bharti V. Evaluation of efficacy of autologous platelet-rich fibrin with demineralized-freeze dried bone allograft in the treatment of periodontal intrabony defects. J Indian Soc Periodontol. 2013; 17:361-366.

9. Hauser F, Gaydarov N, Badoud I, Vazquez L, Bernard JP, Ammann P. Clinical and histological evaluation of postextraction platelet-rich fibrin socket filling: a prospective randomized controlled study. Implant Dent. 2013; 22:295-303.

10. Xuan F, Lee CU, Son JS, Jeong SM, Choi BH. A comparative study of the regenerative effect of sinus bone grafting with platelet-rich fibrin-mixed Bio-Oss and commercial fibrin-mixed Bio-Oss: an experimental study. J Craniomaxillofac Surg. 2014;42(4):e47-50.

11. Bains R, Bains VK, Loomba K, Verma K, Nasir A. Management of pulpal floor perforation and grade II Furcation involvement using mineral trioxide aggregate and platelet rich fibrin: a clinical report. Contemp Clin Dent 2012; 3 (S2):S223-S227.

12. Clipet F, Tricot S, Alno N, ET AL. In vitro effects of Choukroun's platelet-rich fibrin conditioned medium on 3 different cell lines implicated in dental implantology. Implant Dent. 2012; 21:51.

13. Salgado-Filho I, Zanini AS, Mélega JM, Zanini SA, Psillakis JM. Reparação dos traumas de pele e tecidos moles. In:. Cirurgia plástica reparadora e estética. Rio de Janeiro: Medsi; 1992. p. 139-45.

14. Jameson C. Autologus Platelet Concentrate for the production of Platelet Gel. LABMEDICINE. 2007; 38(1): 39-42. DOI: http://dx.doi.org/10.1309/3UA5HWYVKNCE01AR

15. Gupta V, Bains BK, Singh GP, Mathur A, Bains R. Regenerative potential of platelet rich fibrin in dentistry: Literature review. Asian J Oral Health Allied Sci.
2011;1:22-28.

16. Grey RG. Fibrin as a hemostatic in cerebral surgery Surg Gynecol Obstet. 1915; 21:452-454.

17. Clipet F, Tricot S, Alno N, et al. In vitro effects of Choukroun's platelet-rich fibrin conditioned medium on 3 different cell lines implicated in dental implantology. Implant Dent. 2012; 21(1):51-6.

18. García V, Corral I, Bascones A. Plasma rico en plaquetas y su utilización en implantología dental. Avances en Periodoncia e Implantología Oral. 2004;16(2): 81-92.

19. Bennett NT, Schultz GS. Growth factors and wound healing; Part II: Role in normal and chronic wound healing. American Journal of Surgery. 1993;166(1):7481.

20. Stephan EB, Renjen R, Lynch SE, Dziak R. Platelet-derived growth factor enhancement of a mineral-collagen bone substitute. J. Periodontol. 2000; 71:1887-1892.

21. Strayhorn CL, Garret JS, Dunn RL, Benedict JJ, Somernan MJ. Growth factors regulate expression of osteoblast associated genes. J. Periodontol. 1999;70:1345-1354.

22. Alevizopoulos K, Vlach J, Hennecke S, Amati B. Cyclin E and c-Myc promote cell proliferation in the presence of p16NK4a and of hypophosphorylated retinoblastoma family proteins. EMBO J. 1997; 16:5322-5333.

23. Ogata N, Chikazu D, Kubota N, et al. Insulin receptor substrate-1 in osteoblast is indispensable for maintaining bone turnover. J Clin Invest. 2000;105(7):935-43.

24. Choukroun J, Diss A, Simonpieri A, et al. Platelet-rich fibrin (PRF): A second-generation platelet concentrate. Part V: Histologic evaluations of PRF effects on bone allograft maturation in sinus lift. Oral Surg Oral Med Oral Pathol Oral Radiol Endod. 2006; 101:299_ 303.

25. Mazor Z, Horowitz RA, Del Corso M, Prasad HS, Rohrer MD, Dohan-Ehrenfest DM. Sinus floor augmentation with simultaneous implant placement using Choukroun's platelet-rich fibrin as the sole grafting material: A radiologic and histologic study at 6 months. J Periodontol. 2009; 80:2056-64.

26. Simonpieri A, Del Corso M, Sammartino G, Dohan Ehrenfest DM. The relevance of Choukroun's platelet-rich fibrin and metronidazole during complex maxillary rehabilitations using bone allograft. Part I: A new grafting protocol. Implant Dent. 2009;18:10211.

27. Toffler M, Toscano N, Holtzclaw D, Corso MD, Dohan-Ehrenfest DM. Introducing Choukroun's platelet rich fibrin (PRF) to the reconstructive surgery milieu. J Implant Adv Clin Dent. 2009; 1:21-30.

28. Kfir E, Kfir V, Kaluski E. Immediate bone augmentation after infected tooth extraction using titanium 
membranes. J Oral Implantol. 2007; 33:133-8.

29. Anilkumar K, Geetha A, Sudhakar U, Ramakrishnan T, Vijayalakshmi R, Pameela E. Platelet-rich-fibrin: A novel root coverage approach. J Indian Soc Periodontol. 2009;13:50-4.

30. Aroca S, Keglevich T, Barbieri B, Gera I, Etienne D. Clinical evaluation of a modified coronally advanced flap alone or in combination with a platelet-rich fibrin membrane for the treatment of adjacent multiple gingival recessions: A 6-month study. J Periodontol. 2009;80:244-52.

31. Kanakamedala A, Ari G, Sudhakar U, Vijayalakshmi R, Ramakrishana T, Emmadi P. Treatment of a furcation defect with a combination of platelet rich fibrin and bone graft-A case report. ENDO (Lond Engl). 2009; 3:127-35.

32. Sandhu GK, Khinda PK, Gill AS, Kalra HS. Surgical re-entry evaluation of regenerative efficacy of bioactive Gengigel( $\left.{ }^{\circledR}\right)$ and platelet-rich fibrin in the treatment of grade II furcation: A novel approach. Contemp Clin Dent. 2015; 6(4): 570-3.

33. Shivashankar VY, Johns DA, Vidyanath S, Sam G. Combination of platelet rich fibrin, hydroxyapatite and PRF membrane in the management of large inflammatory periapical lesion. J Conserv Dent. 2013; 16:261-264.

34. Jayalakshmi KB, Agarwal S, Singh MP, Vishwanath BT, Krishna A, Agrawal R. Platelet-rich fibrin with beta-tricalcium phosphate-a noval approach for bone augmentation in chronic periapical lesion: a case report. Case Rep Dent. 2012; 2012: 902858.
35. Keswani D, Pandey RK. Revascularization of an immature tooth with a necrotic pulp using platelet-rich fibrin: a case report. Int Endod J. 2013; 46:10961104.

36. Bains R, Bains VK, Loomba K, Verma K, Nasir A. Management of pulpal floor perforation and grade II Furcation involvement using mineral trioxide aggregate and platelet rich fibrin: a clinical report. Contemp Clin Dent. 2012;3(S2): S223-S227.

37. Shivashankar VY, Johns DA, Vidyanath S, Kumar MR. Platelet rich fibrin in the revitalization of tooth with necrotic pulp and open apex. J Conserv Dent. 2012;15:395-398.

38. Rudagi KB, Rudagi B. One-step apexification in immature tooth using grey mineral trioxide aggregate as an apical barrier and autologus platelet rich fibrin membrane as an internal matrix. J Conserv Dent. 2012;15:196-199.

39. Suresh N, Chandrasekaran B, Muthusamy S, Kannan S, Muthu K. Application of platelet rich fibrin for management of an electrosurge induced osteonecrosis involving maxillary alveolus. Singapore Dent J. 2015;36:39-43.

Recibido : 30/12/2015

Aceptado: 21/06/2016 